\title{
La enseñanza de procedimientos en el laboratorio de ciencias de formación docente
}

Silvia Umpiérrez Oroño*

María Teresa Cafferata**

"La enseñanza como actividad práctica se realiza siempre a la luz de alguna concepción sobre lo deseable y lo posible."

Edith Litwin, 1997.

\section{Resumen:}

Este trabajo presenta una investigación cuyos objetivos fueron conocer qué tipos de procedimientos enseñan los profesores de formación docente en el laboratorio de ciencias; cómo los enseñan y qué elementos modelan sus intenciones y acciones.

Fue una investigación de carácter cualitativo, en la que intentamos la comprensión e interpretación de buenas prácticas de enseñanza.

El análisis de los datos, propuesto como la construcción de una escultura, permitió generar categorías desde tres dimensiones: El escenario de la escultura, considera los factores externos que modelan la enseñanza de procedimientos en el laboratorio, produciendo dos categorías; La inspiración del escultor, mira las concepciones de los profesores, originando cuatro categorías y La obra final: esclavos emuladores y patrones supervisados interpreta cómo se enseñaron distintos procedimientos, generando dos categorías.

Finalmente reflexionamos sobre repercusiones que podría tener la Teoría de Sistemas en la enseñanza de procedimientos y en la investigación educativa.

\begin{abstract}
:
The present work presents some aspects of a research whose objectives were to know what kind of scientific processes do professors teach at the science laboratory in an institution that trains future secondary school teachers; how do they teach such processes and which elements shape their actions and intentions.

It was a qualitative study during which we tried to understand and to interpret good teaching practices

The data analysis, proposed as a sculpture construction, let us generate categories from three dimensions: the Sculpture stage that considers the external factors that model the processes teaching at the laboratory, produced two categories; The Sculptor inspiration that looks into professors believes, generated four categories; The finished work: emulous slaves and supervised masters, interprets how different processes are taught, producing two categories

Finally, we reflect on the impacts the Theory of Systems would have on the scientific processes teaching and on educative research.
\end{abstract}

\section{I ntroducción:}

El trabajo de investigación en el que se basa este artículo, fue realizado como Tesis de la Maestría en Educación. Sus objetivos fueron conocer qué tipos de procedimientos enseñan los profesores de formación docente en el laboratorio de ciencias; cómo los enseñan y qué elementos modelan sus intenciones y acciones. 
Eran objetivos sumamente concretos y tangibles, nos hacían sentir muy seguras, y nunca sospechamos, sino casi hasta el final del trabajo, adónde nos iban a conducir. Pero, como dice S.J. Gould (1993), toda metodología científica de calidad debe contener "una enseñanza que se eleva por encima de todas las otras, en lo que concierne a las reglas de procedimiento". Así que los resultados finales nos hicieron ir más allá, en un proceso de aprendizaje personal que consideramos fue apreciable y válido para ser compartido en esta publicación.

\section{Consideraciones previas o la chispa inicial:}

Cuando intentamos reconstruir las causas de porqué elegimos trabajar sobre la enseñanza de procedimientos (o contenidos procedimentales como algunos autores los llaman), surgen respuestas en relación con una de las dimensiones del conocimiento científico, la procedimental, muy presente en la enseñanza de las ciencias en el laboratorio. Cuando queremos justificar porqué elegimos trabajar en el laboratorio, surgen respuestas tales como: es el lugar donde se enseñan procedimientos por excelencia. Nos adentramos en un ciclo de laboratorioprocedimientos-laboratorio que nos rememora el eterno dilema del huevo y la gallina.

En primer lugar, siempre hay una chispa inicial, que cuando inicia un incendio, la dejamos de ver. Esa chispa inicial fue una inquietud que surge más bien desde el rol docente cotidiano y no desde la postura de investigación que asumimos luego. Son "los datos no elaborados" al decir de Goodson y Walker (1995), que pueden contribuir a definir o esclarecer un problema (en este caso el del huevo-gallina, o el del laboratorio-procedimientos). La chispa consistió en un llamado de atención sobre los gastos en equipamientos de laboratorio, bastante significativos con relación al presupuesto total de inversión de una institución educativa; dichos gastos, en proporción a los frutos, parecen poco provechosos para algunos autores y varios de nuestros colegas.

Insausti y Merino (2000) afirman que los trabajos de investigación en los últimos 10 años, han mostrado un panorama pobre en en cuanto a los resultados educativos obtenidos a partir de trabajos prácticos tipo "receta", caracterizados fundamentalmente por una falta de planificación, creatividad, indagación y formulación de hipótesis por parte de los alumnos. Millar et al. (1999) afirman que, a pesar de que el trabajo práctico en ciencias es el que los alumnos afirman disfrutar más, no se logra que aprendan aquello que los docentes desean o se han propuesto. De Pro Bueno (1995) marca la realidad de que no se ha propiciado lo suficiente el laboratorio como ámbito de enseñanza de todos los procedimientos, sino más bien aquellos menos complejos, como manipular, observar y medir.

En segundo lugar, tenemos que aclarar que cuando hablamos de procedimientos o contenidos procedimentales nos estamos refiriendo no sólo a destrezas y técnicas manipulativas, sino también a aquellas estrategias de investigación y procesos cognitivos que se ponen en juego en la producción y reconstrucción del conocimiento. Concordamos con Salgueiro (1998) en que la clasificación de los contenidos en conceptuales, actitudinales y procedimentales, es un artificio "de naturaleza pedagógica" pero en el momento de enseñar, deben abordarse todas las perspectivas. Lo que puede observarse en realidad durante la clase, no es una separación o una integración de los diversos tipos de contenidos todo el tiempo, sino que más bien en ciertos momentos o lugares priman unos sobre otros. La artificiosidad de dividir los contenidos en varios tipos, es una construcción intelectual que puede ayudar a comprender el pensamiento y el comportamiento de las personas; se ha creado para contribuir a vislumbrar los procesos cognoscitivos y conductuales, para analizar de forma fragmentada, lo que en realidad se da de manera integrada (Zabala 2000); creemos también, que es un llamado de atención sobre el riesgo de despojar al conocimiento de las dimensiones que lo constituyen reduciéndolo, como frecuentemente sucede en las aulas, a su dimensión conceptual. 
En tercer lugar, la enseñanza de procedimientos se lleva a cabo frecuentemente por medio de prácticas de enseñanza denominadas "trabajos prácticos". Y los trabajos prácticos, en las instituciones educativas, se llevan a cabo generalmente (no exclusivamente) en los laboratorios. Cafferata (2002) define a las prácticas en el laboratorio, como "aquellas actividades de enseñanza que tienen lugar en el laboratorio o en el aula, durante las cuales los participantes no sólo manipulan y observan materiales, sino también se involucran intelectualmente usando y aplicando conceptos teóricos". Hodson (1994) afirma: "...existe la suposición de que el trabajo práctico equivale necesariamente a trabajar sobre un banco de laboratorio y que este tipo de trabajo siempre incluye la experimentación. Yo pienso que cualquier método de aprendizaje que exija a los aprendices que sean activos en lugar de pasivos concuerda con la idea de que los estudiantes aprenden mejor a través de la experiencia directa, por lo que podría ser descrito como trabajo práctico."

Considerando lo que hemos desarrollado en los párrafos anteriores, elegimos centrar nuestro estudio en los procedimientos de las Ciencias Naturales que se enseñan principalmente bajo determinada modalidad: el trabajo práctico, para lo cual, debíamos concurrir al laboratorio. Y nos preguntamos: ¿Qué enseñan los profesores en el laboratorio? ¿Cómo influyen sus concepciones, su experiencia laboral, su historia profesional en dicha enseñanza? ¿Cómo se usan los materiales didácticos? ¿Cómo organizan la actividad en él? ¿Qué lugar dan al trabajo en el laboratorio en la formación académica y profesional de sus estudiantes, futuros profesores?

\section{La práctica docente.}

Nos respaldamos básicamente en Feldman (1999) para trabajar desde la enseñanza. Este autor define la enseñanza como un campo de propósitos, y frente a la pregunta de si existe enseñanza si no hubo aprendizaje, responde "La enseñanza no se define por el éxito del intento sino por el tipo de actividad en la que ambos sujetos se ven comprometidos. La enseñanza expresa propósito (promover el aprendizaje) y no logros". Entonces ya desde el inicio comenzamos a jugar con la trascendencia de las intenciones del profesor: éstas definen su rol docente.

La metodología de enseñanza es una construcción compleja. Díaz Barriga (1995) afirma que sólo desde la especificidad del contenido y una "posición interrogativa ante él, es posible superar la postura instrumentalista en relación con el método". Se plantea, entonces, una visión más compleja, para la cual la metodología de enseñanza no es un encadenamiento preestablecido de técnicas, sino una construcción, atendiendo el "problema de cómo abordar el objeto en su lógica particular a partir de las peculiaridades del sujeto que aprende". Es una construcción "de carácter singular, que se genera en relación con un objeto de estudio particular y con sujetos particulares", docente y alumno, en un contexto determinado.

El concepto que tiene el docente de ciencias acerca de qué es ciencia y cómo se trabaja científicamente, incide en sus construcciones metodológicas. La planificación, la acción y la evaluación de aula, se basan fundamentalmente en aquello en lo que el profesor cree, en lo que sabe y en lo que se propone enseñar. El docente de ciencias construye su práctica a partir de la interacción de su concepción de ciencia, sus conocimientos disciplinares y sus conocimientos pedagógicos, contextualizados a un nivel, a una escuela, a sus referencias axiológicas (su sistema de valores, su ideología, sus concepciones de educación) y su historia.

La formación y la experiencia también marcan la práctica docente. Lo que el profesor hace está condicionado por su idea acerca de cómo se construyen los saberes. "No es posible enseñar nada -nos dice Zabala (2000)- sin partir de una idea de cómo se producen los aprendizajes."Su formación y sus experiencias de aula tanto como alumno o como profesor en 
otros contextos, le han ido proporcionando una concepción de los procesos de construcción de aprendizaje única e irrepetible. Y esa concepción modela sus estrategias didácticas actuales. Por eso cada docente tiene una generalidad en sus prácticas, similar a otros con los que comparte la asignatura, o el nivel, pero tiene una singularidad dada por su historia.

\section{Pero por sobre todo, la buena práctica}

Decidimos investigar buenas prácticas de enseñanza siguiendo lo que proponen varios autores, principalmente Litwin (1997), quien enfatiza la recuperación de la ética y los valores en la enseñanza, a lo cual adherimos profundamente. También nos basamos en Feldman (1999), quién afirma que una enseñanza es buena, entre otras cosas, si el docente realiza todos los esfuerzos posibles para que los alumnos aprendan. Un buen docente, también, es aquel que logra abrir muchas entradas al mismo concepto para sus alumnos (Litwin 1997; Fernández 1999).

Acordamos en definir como una buena práctica, aquella en la que se destaca la capacidad de despertar en el docente y en el alumno un proceso reflexivo y crítico; de fomentar el "pensar" en la clase; de favorecer el reconocimiento de los problemas que surgen cuando los alumnos resuelven las tareas; de mostrar a los estudiantes los procesos del pensar característicos de las actuaciones expertas.

Pero las buenas prácticas deben definirse también y sobre todo, por los aspectos morales y epistemológicos, por la recuperación de la ética y los valores, por las actitudes, las conductas y las maneras de vincularse con los alumnos en la clase (Litwin 1997).

\section{El marco metodológico:}

Encuadramos la investigación en un marco cualitativo. Desde el inicio asumimos las características del abordaje cualitativo como riesgos: es subjetivo; frecuentemente genera más preguntas que respuestas; sus contribuciones son humildes y aparecen a un ritmo parsimonioso, por lo que su aporte al avance de la práctica social es lento; puede llegar a ser tendencioso; tiene altos riesgos éticos; sus costos en tiempo y dinero pueden ser elevados (Stake 1999). Sin embargo, analizando estos "riesgos", no se puede afirmar que el abordaje cuantitativo esté totalmente libre de ellos. Es de destacar que el hecho de generar más preguntas que respuestas puede llegar a ser iluminador de campos por explorar, y abrir camino a nuevos investigadores y trabajos científicos.

\section{Dónde, quiénes y cómo:}

Los datos se recabaron en un centro de formación docente para educación media, del interior del Uruguay. A partir del año 2004, se realizaron observaciones pilotos durante tres meses. Luego en el año 2005, se comenzó el trabajo de campo en el mes de junio, que se prolongó hasta el mes de diciembre. El análisis culminó en el mes de marzo del año 2006.

En este trabajo se utilizaron las siguientes herramientas para la recolección de datos, que serán detalladas más adelante: observación de clases, entrevistas, cuestionarios escritos a los alumnos y documentos que facilitaron los docentes (planificaciones, evaluaciones, etc.).

El uso de más de una técnica de recogida de datos, fue una manera de incluir en los resultados el punto de vista del otro (Stake 1999; Woods 1993; Valles 1997; Goetz y Le Compte 1988), y contribuir a la validación interna y externa. Fue también una forma de que el trabajo reflejara las realidades múltiples: pretendimos lograr una multidimensionalidad en la visión final del fenómeno estudiado en esta propuesta. 
Trabajamos con cinco docentes: tres de biología y dos de química. En general es aconsejable trabajar sobre una muestra "intencional, sistemática y teóricamente conducida", pero esto a veces se dificulta por problemas de acceso (Woods 1993). No se trató de una muestra representativa, sino de una selección de casos idóneos y asequibles a la vez. Se realizó una "selección estratégica" (Valles 1997), en la cual la preocupación no se centró en la representatividad, sino en: la heterogeneidad, manteniendo equilibrio entre "la variación y la tipicidad"; la selección de contextos personales, relevantes al tema; y la accesibilidad. Elegimos cinco docentes con diferentes procedencia formativa (formación en investigación, formación docente o ambas), y distinta experiencias anteriores en otros sistemas educativos o científicos. A su vez, ellos tenían en común: la antigüedad en la institución (entre 5 y 6 años), y el área de trabajo (Ciencias de la Naturaleza). Eran buenos docentes en el sentido moral y pedagógico, que consideraban la enseñanza en el laboratorio como un aspecto relevante en la enseñanza, y que habían demostrado, en palabras y acciones, que creían importante la investigación educativa como una forma más de mejorar la calidad de la educación.

En principio nos habíamos propuesto realizar tres observaciones a cada docente, pero en realidad se llevaron a cabo un número variable de las mismas, totalizando ocho. Esto se debió a las oportunidades que se dieron conciliando tiempos, trabajo y disponibilidades de horarios.

Hubo un caso especial de una docente a la que no se le realizó ninguna observación de clase, debido a que, si bien cumplía con los requisitos de "buena docente", no concurrió al laboratorio durante el período en el cual estuvimos recogiendo los datos. Si bien podía parecer en un primer momento que esta docente no era la más indicada, ya que concurría al laboratorio con baja frecuencia (como ella misma lo expresó en la entrevista), resultó importante porque pudimos realizar una indagación acerca de porqué -a pesar de considerar de relevancia el trabajo en laboratorio-, concurría con baja asiduidad. Esta entrevista resultó enriquecedora para este trabajo, ya que aportó una perspectiva distinta de las limitantes de los docentes en el momento de elegir cómo y dónde enseñar. No es fácil hallar un profesor que sea capaz de reconocer las falencias o aspectos mejorables de su propio trabajo, frente a la investigadora que indaga justamente sobre aquello que el docente cree que es una limitación personal.

En total se realizaron ocho entrevistas focalizadas y cinco entrevistas finales. Las entrevistas fueron fundamentales en el momento de ampliar la comprensión de lo observado, de validar las interpretaciones de la intención del docente en clase, de multidimensionar la visión del fenómeno en estudio. En primer lugar, se pretendía validar internamente lo observado en el aula. Este tipo de entrevistas son referenciadas por Pardinas (1999) y Valles (1997) como "focalizadas". En segundo lugar, a través de las entrevistas finales a cada profesor, se pretendía conocer otros aspectos más globales, conducentes a la comprensión de la práctica docente en el laboratorio, y relacionarla con su historia, el contexto educativo, disciplinar e institucional; su concepción de ciencia, de enseñar y de aprender procedimientos; su concepción del trabajo práctico en el laboratorio.

Se le solicitó a los docentes todo aquel material escrito que estuvieran dispuestos a compartir, por ejemplo evaluaciones, planificaciones, etc. Se realizó un breve cuestionario escrito a los alumnos que habían participado de las clases que fueron observadas.

Se enviaron los avances del análisis a los profesores, solicitándoles que dieran su opinión acerca de las categorías creadas y las interpretaciones que las conformaban. De los cinco docentes, tres opinaron que estaban de acuerdo, sin realizar aportes o preguntas, mientras que dos de ellos realizaron puntualizaciones, complementos, o preguntas orientadoras. 
El análisis se basó en la estrategia denominada "método comparativo constante" (Taylor y Bogdan 1987), en la que "mediante la comparación de incidentes específicos de los datos", el investigador integra sus resultados generando una teoría, que los contiene y les da coherencia. Se produjo una estratificación, comenzando con un análisis preliminar que se superpuso a la colecta de datos. Esta primera instancia interpretativa se constituyó en el "análisis especulativo" (Woods 1993). Este primer análisis ayudó a ir familiarizándonos con los datos desde un principio. Nos permitió ir realizando un proceso simultáneo de colectar datos y repensar una y otra vez sobre el desarrollo del trabajo. Esto se realizó por un "espiral de comprensión" en el que las acciones se intensificaron yendo una y otra vez "hacia atrás y hacia adelante entre la observación, el análisis y la comprensión" (Lacey 1976, citado por Woods 1993).

Luego de terminada la recogida de datos, comenzó una nueva fase del análisis. Fue lo que Woods (1993) denomina "clasificación y categorización": "significa ordenar los datos de una forma coherente, completa, lógica y sucinta". A medida que el trabajo avanzaba, algunas categorías que habían sido creadas, desaparecieron, otras se unieron, y aparecieron nuevas.

Una vez agrupadas las partes de las transcripciones correspondientes a cada categoría, se ampliaron y redactaron las interpretaciones, haciendo referencia también a datos bibliográficos hallados por otros investigadores, y relacionadas con las mismas. Como forma complementaria de análisis se realizó una caracterización formal de los prácticos de cada docente, basada en el mapeo de trabajos prácticos propuesto por Millar et al. (1999).

Finalmente se realizó un cruzamiento de todos los datos, como una forma de ampliar la comprensión y de llevar a cabo la triangulación. En esta etapa se añadieron al análisis los cuestionarios de los estudiantes, los documentos de los docentes y sus comentarios acerca de las categorías.

\section{El nudo central del trabajo:}

Comenzamos a buscar recurrencias, vínculos, correspondencias, a partir de los datos. Se dio una construcción basada en las acciones y en las palabras de los docentes. Buscamos un sentido por medio del cual generar una teoría que los contuviera y que les diera coherencia. Recurrimos a la lectura de los avances de otros investigadores y de los propios docentes participantes, hasta que vislumbramos una figura final, con colores que delineaban una estructura y un entorno. Nos propusimos entonces, una mirada a la construcción de la clase como la creación de una escultura artística. Desde la dimensión El escenario de la escultura, analizamos los factores extrínsecos que modelan la enseñanza de procedimientos en el laboratorio; desde La inspiración del escultor, los factores intrínsecos; y desde la dimensión La obra final: esclavos emuladores y patrones supervisados, analizamos cómo se enseñaron distintos tipos de procedimientos.

Desde nuestra primera dimensión, El escenario de la escultura, analizamos entonces los elementos externos al docente (el contexto) que modelan la enseñanza de procedimientos en el laboratorio. Cuando hablamos de la influencia del contexto en la enseñanza de procedimientos, nos referimos a dos caras de éste: el contexto curricular y el contexto físico. Encontramos dos categorías: "Superando contextos" y "Ustedes como docentes".

"Superando contextos" abarcó por un lado el contexto temporal, el cual implica un compromiso entre los tiempos que se pasan en el aula común y en el laboratorio; y por otro el contexto físico, que se trata de los espacios y recursos disponibles en el laboratorio.

\section{4 - Universidad ORT Uruguay}


Dentro de esta categoría diferenciamos dos subcategorías: "T / P ¿Tiempo / Programa o Teórico / Práctico?" y "Trabajando con lo que 'no tenemos' ".

En la primera subcategoría hallamos que los docentes consideraron fundamental ir al laboratorio, manteniendo un equilibrio cuidadoso para lograr conciliar intereses con los objetivos del programa. Aunque el trabajo práctico "lleva más tiempo" es considerado un elemento de motivación, que posibilita la creatividad, la aplicación de contenidos vistos desde lo teórico, la perspectiva de utilidad de los conocimientos. Se juega con "compromisos" que el docente preasume con el programa, y con las posibilidades educativas que éste considera que brinda el trabajo práctico en el laboratorio.

En la segunda subcategoría, "Trabajando con lo que 'no tenemos' ", el hallazgo más importante fue que los docentes no dejan de enseñar ante la carencia de recursos. Se las ingenian, y también utilizan la falta de algunos materiales para enseñar a trabajar en contextos diferentes.

La segunda categoría, "Ustedes como docentes", muestra cómo el contexto institucional se introduce en el aula (metafóricamente) en forma de un alumno liceal omnipresente. El trabajo en el laboratorio contempla siempre a un tercero, que es el futuro alumno. Los profesores hacen mucho énfasis en la planificación, vinculándola con cuestiones de aprovechamiento del tiempo y la responsabilidad de manejar adecuadamente los materiales. Se destaca la insistencia en la planificación como un procedimiento intelectual relevante para los futuros docentes. No pueden ir a clase sin estar seguros de qué es lo que van a necesitar, de cómo van a trabajar. Poner a punto las técnicas de trabajo es uno de los aspectos más importantes de una planificación.

Podemos decir, resumiendo, que los factores externos al docente no son los que tienen la mayor influencia en estas prácticas pues actúan modelando las estrategias didácticas en términos generales. No queremos decir que no impactan, sino que tienen menor repercusión que otros factores en las decisiones de los docentes.

Esto podría deberse a dos causas. La primera, se relaciona con la institución, que no tiene grandes limitantes de espacio y de recursos. Está medianamente bien equipada en cuanto a laboratorios, materiales y equipos. Cuando faltan algunas cosas, esto no resulta un obstáculo para los docentes que se las arreglan para remediarlas. La segunda, podría ser que los profesores tienen libertad de cátedra. No se imponen, desde lo institucional, pautas detalladas sobre qué enseñar o cómo enseñar. Esto quiere decir que los profesores deciden con bastante autonomía cómo van a trabajar.

La segunda dimensión, La inspiración del escultor, hace referencia a factores intrínsecos a los profesores, básicamente sus concepciones. Hallamos que esos factores tienen mayor impacto que los extrínsecos, sobre la enseñanza de procedimientos en el laboratorio. Caracterizamos a continuación las cuatro categorías encontradas.

Tal vez Juan Pérez no aprende como yo" nos ubica frente a docentes que consideran que todos aprendemos de manera diferente, y que por lo tanto hay un deber moral de enseñar de formas diversas también. Se apuesta a la diversidad de abordajes, no sólo desde las posibilidades que brinda el trabajo en el laboratorio, sino que además dentro del laboratorio se ponen en juego diversas estrategias de enseñanza. 
"Ciencia y didáctica”, se relaciona con la frase de Anahí Mastache (1998): “...ninguna construcción didáctica es neutral respecto a la filosofía de la ciencia que le subyace". Esta categoría da cuenta de las relaciones halladas entre las estrategias de enseñanza utilizadas y el desarrollo científico. Existe un entramado sutil entre lo que es la concepción de ciencia del docente, los objetivos que tiene en mente cuando va a enseñar procedimientos científicos a los futuros docentes y la forma en que propone hacerlo. En esta categoría, hallamos dos subcategorías.

En la primera, "Viejas recetas, nuevas propuestas", hallamos a los docentes manifestando explícitamente que quieren dejar atrás las metodologías conductistas, la aplicación del método científico. Se proponen trabajar desde el constructivismo, con propuestas que den libertad de acción y de creación al estudiante, como persona única y diferente en sus capacidades. Observamos algunas instancias en las que se invoca la memorización, o se cuela algún resabio del principio de correspondencia del asociacionismo; por ejemplo apelar a la demostración "fiel de la realidad" para que el estudiante la "reproduzca". Pero retomamos que hay una reflexión muy válida, que priman más las estrategias del constructivismo: propuestas abiertas, autogestionadas y orientadas, con una mirada al proceso más que a los resultados, con una reflexión mediando el uso de los recursos didácticos.

La segunda subcategoría, "Del tubo de ensayo a la pileta de la cocina", alude a la concepción social del científico, que ha cambiado, y se nos presenta como más cercano, más común, más humano. Vemos cómo los docentes acercan los contenidos procedimentales científicos que se enseñan en el laboratorio, a aspectos que tienen que ver con la vida cotidiana, la salud, las actitudes y valores humanos.

"El que se equivoca, gana" es una categoría que surge del manejo del error por parte de los docentes. Si bien se puede utilizar el error como una estrategia didáctica, sustituyendo un enfoque de resultados por uno de procesos, hallamos diversas actitudes frente al error. Algunos profesores no lo toleran. Otros lo toleran pero no lo aprovechan. Y otros son capaces de enseñar a partir del error. El miedo a equivocarse de algunos docentes, demostró ser un obstáculo mucho mayor que el error en sí. Los profesores se sienten expuestos en el laboratorio, más cuando se proponen estrategias de trabajo abiertas. Algunos de ellos no concurren con más asiduidad al laboratorio por miedo a equivocarse, optando por no sacar provecho de sus errores. El no saber manejar el propio error, también obstaculiza el manejo de los errores de los alumnos. Como dicen De la Torre y Barrios (2000), el error no puede ser un fin en sí mismo, no se puede ir al laboratorio sin estar seguros de lo que se va a hacer, y no se puede provocar el error en los alumnos para trabajar a partir de él. El error es un emergente natural en la clase, y hay que prepararse para usarlo didácticamente cuando aparece.

La cuarta categoría, "Tocar el piano con una sola mano", nace tomando la frase textual de una entrevista a uno de los profesores. Este docente afirma que enseñar sólo desde lo conceptual, sin lo procedimental, es un acto de enseñanza incompleto. Tomamos también la frase de Mastache (1998) para resumir los hallazgos de esta categoría: "La práctica no es vista como una simple aplicación de la teoría, sino como una instancia de validación y reconstrucción de la misma". El trabajo práctico en estas clases no sustituye, sino que suma. Suma en motivación, en resignificación de conocimientos, en oportunidades de enseñar diferente, y ofrece recursos que no se encuentran en otros ámbitos.

Finalmente, la tercera dimensión la denominamos La obra final: esclavos emuladores y patrones supervisados. Haciendo alusión al nombre de la dimensión, parecería acertado recordar que emular quiere decir: imitar las acciones de otro procurando igualarle y aún excederle.

\section{6 - Universidad ORT Uruguay}


Comenzamos este tramo final con la cita de un libro que ya tiene más de cien años, es el cual hallamos correspondencia con nuestras interpretaciones:

"Los experimentos tienen un múltiple objeto. (...) Mostrarle la universalidad de las leyes naturales, que no se muestran sólo al conjuro de los hermosos aparatos de laboratorio, en los que brilla el bronce y el cristal, sino también en las humildes máquinas que ha construido con sus propias manos; convencerle de que esas fuerzas le cercan, le rodean, le esclavizan, pero que también le ofrecen ocasión de esclavizarlas, de cercarlas a su vez (...) Todo esto es muy difícil de hacer, de manera que no deberemos desanimarnos si (...) no sale bien la primera vez. Haremos varios ensayos, y luego notaremos, con satisfacción, que los errores son cada vez menores." Ernesto Nelson 1905. Primeros ensayos en la física y en la química. Chicago: Editorial D. Appleton y Cía.

Desde esta dimensión vemos cómo los docentes siguen un camino de enseñanza de procedimientos que va desde métodos que se acercan al conductismo y al aprendizaje por asociación hasta posturas más innovadoras. Esas dos estrategias no aparecen ligadas al azar en cuanto al tipo de contenido que se está enseñando ni al aspecto temporal. Hay una correlación que analizamos y validamos en las categorías que describimos a continuación.

"Haz lo que yo digo y también lo que yo hago", se vincula con la denominada tradición mimética de Jackson (2002), aplicada, en este caso, a la enseñanza de procedimientos manipulativos. Los docentes acompañan sus demostraciones con un discurso instructivo, luego piden a los estudiantes que lo imiten, y que finalmente lo repitan, hasta que el procedimiento que están aprendiendo sea bien ejecutado.

Los docentes consideran que los procedimientos pueden ordenarse desde los más simples manipulativos, hasta los más complejos, los intelectuales. Deben ser enseñados en ese orden, ya que los primeros posibilitan la enseñanza de los otros. El aprendizaje asociativo es un preámbulo necesario para el aprendizaje constructivo.

La segunda categoría, "La emancipación", muestra cómo los procedimientos intelectuales se enseñan de una manera diferente a los manipulativos. Se impulsa a los estudiantes a "no copiar", a ser creativos, a autogestionar su aprendizaje.

Siguiendo a Sanmartí et al. (1990) podemos distinguir los tipos de procedimientos científicos (intelectuales y manipulativos) como: pre-experimentales, experimentales, y postexperimentales. Los procedimientos pre-experimentales son los que "corresponden a la etapa creativa, de definición y planificación de la empresa: enunciar el problema, identificar las variables, construir hipótesis, planificar los experimentos". Los experimentales son los que se aplican durante la ejecución misma de la tarea: "observar, recoger datos (...) a partir de manipulaciones diversas". Los post-experimentales tienen que ver con la "evaluación y comunicación de los resultados".

Los docentes observados ponen en juego todos estos tipos de procedimientos científicos en el laboratorio, con diferente frecuencia. Sostienen que el planteamiento de hipótesis, la resolución de problemas y el diseño de experiencias, son los contenidos científicos procedimentales intelectuales más complejos para enseñar en relación con los prácticos de laboratorio.

Cuando los alumnos son impulsados a trabajar de forma autónoma en la fase pre-experimental para, entre otras cosas, estimular su creatividad, se les incita a que sean independientes, diseñen trabajos, planteen hipótesis, las comprueben o las rechacen. Es un trabajo supervisado 
por los docentes. "Convendría tener en cuenta que no parece que los hechos vayan a tener para el alumno un significado unívoco" (Carretero 1996). La comprobación de hipótesis a partir de trabajo autogestionado por los estudiantes, va acompañada, en estas clases, por un equilibrio entre el dejarlos hacer y la instrucción. Los contenidos procedimentales intelectuales requieren de actividades expositivas por parte del docente. " ... los estudios actuales muestran que existen diferentes métodos instruccionales que resultan eficaces a la hora de optimizar las habilidades de razonamiento tanto de adolescentes como de adultos" (Carretero 1996). Consiste, en nuestros casos, en un acompañamiento de la autogestión, con explicitación de algunos aspectos que el docente, con todo su bagaje conceptual, puede resaltar para reorientar el trabajo de los estudiantes, o enriquecer los resultados. No consiste en contarles el "final del cuento" sino en ayudarlos a ir dando vuelta las páginas. Los estudiantes se transforman así en patrones de sus propios aprendizajes, bajo la supervisión de los profesores. Este estímulo de libertad va acompañado de orientación bajo diferentes formas, de manera que se generaron tres subcategorías: "Preguntas obvias con respuestas no tan obvias...", "La metacognición en el laboratorio" y "Tres maneras de enseñar a diseñar".

En la primera, una de las profesoras parte de las ideas e intereses de los estudiantes, realizando preguntas que los hacen replantearlas, dudar, reflexionar, ponerlas a prueba. Es consciente de que un mismo hecho o proceso observado puede tener más de una interpretación, y como los estudiantes manejan menos cantidad de teoría establecen menos vínculos. La profesora considera que los alumnos necesitan ser orientados para lograr que sus reflexiones, razonamientos, o respuestas, se acerquen a sus objetivos didácticos.

En la segunda subcategoría, hallamos a otra de nuestras docentes razonando en voz alta frente a sus alumnos. Dice Meirieu (1997): "un dispositivo didáctico no se genera por la definición de un objetivo, sino por la hipótesis de la operación mental necesaria para alcanzarlo". La profesora toma una actitud descentralizadora de análisis de los propios procesos cognitivos, y lo hace en voz alta, como una manera de mostrar a los alumnos una forma de resolver un problema, o de analizar un fenómeno.

En la tercera subcategoría, "Tres maneras de enseñar a diseñar", nos adentramos en la descripción de tres maneras de trabajar este contenido procedimental intelectual. Una profesora intenta alejarlos de la visión ingenua y simplificada de innovar, haciendo diseñar los trabajos prácticos a los propios alumnos; una experiencia que parece un salto cognitivo demasiado amplio para estos estudiantes. Otra, lleva a cabo en el laboratorio una experiencia diseñada por uno de los alumnos, y a partir de ahí se proponen alternativas y críticas al trabajo. Un tercer profesor, trabaja a partir del estudio de varias técnicas. Luego eligen una, la llevan a cabo en el laboratorio, y finalmente escriben el protocolo. Más que diseñar una experiencia, diseñan un protocolo. Son entonces tres formas distintas, en las que el común denominador es hacer vivenciar al alumno la experiencia de innovar por medio del diseño de actividades prácticas.

\section{Consideraciones finales:}

Todo parecía girar alrededor del rol docente. El trabajo en el laboratorio estuvo muy marcado por el hecho de estar formando futuros docentes: por énfasis en la planificación, en la prevención de accidentes, en pensar en cómo trabajar en ese otro contexto que los espera cuando egresen. Creemos que se ha dejado en un segundo plano la posibilidad de usar el laboratorio como ámbito generador de conocimientos. Se postergó el rol del docente investigador, generador de teoría y de recursos didácticos. Se miró más al futuro alumno que al presente, es decir que faltó más metacognición acerca del propio proceso de aprendizaje de procedimientos. 
Se logró una muy buena integración entre el trabajo práctico y el teórico. El mundo de las ideas fue puesto en relación con el mundo de los objetos. El "saber hacer" no se redujo a la manipulación. Más que a comprobar, se iba al laboratorio a materializar ideas: el trabajo en el laboratorio no sustituía, sino que sumaba. Al decir de Aparicio (1995) y de Confucio, hace 2500 años, los contenidos conceptuales se olvidan si no se usan, así que hay que enseñar procedimientos de forma ineludible. Hubo una buena articulación del trabajo preexperimental, experimental y postexperimental, mediante el uso integrado de ambos ámbitos: el aula común y el laboratorio.

\section{Y yendo un poco más allá...:}

Hubo diferentes momentos y elementos definitorios. Una inquietud inicial "inocente": ¿se justifica, desde el punto de vista educativo, el gasto relativamente alto en equipos de laboratorio? Como una primera muestra de las "perlas" que fuimos hallando, citamos parte de uno de los cuestionarios que realizamos a los alumnos:

Pregunta: ¿Qué has aprendido en las prácticas de laboratorio de ciencias?

Respuesta: ... he aprendido que a pesar de contar con muy poco material se pueden lograr cosas muy buenas.

Creemos haber cumplido los objetivos planteados. Queríamos y pudimos descubrir en los docentes qué procedimientos enseñaban, cómo lo hacían, y cuáles son los factores que incidieron sobre sus intenciones y acciones en clase. Pero al final del trabajo se abrió un aspecto nuevo, inesperado, fascinante, que consideramos es el más relevante de esta investigación: una nueva pregunta. ¿Cuáles eran esas intenciones? ¿Podríamos acercarnos a conocerlas y describirlas?

\section{Las propiedades emergentes:}

El funcionamiento de un sistema no depende de las cualidades de sus partes particulares, sino que posee una serie de propiedades nuevas, que no se extraen de la adición de las cualidades particulares de sus componentes, sino que sólo aparecen frente a la combinación e interacción de las mismas para dar lugar al sistema: esas son las propiedades emergentes. El concepto de propiedades emergentes, surge en el seno de la Teoría General de Sistemas, y dentro de éste, bajo el paradigma de la complejidad. Dicho paradigma consistente en una propuesta de reforma general del pensamiento científico, estableció, a grandes rasgos, los siguientes principios (Romero 2006):

“- conocer para hacer; es decir, combinar los conocimientos teóricos con los de acción;

- conocer para innovar; (...)

- conocer para repensar lo conocido o pensado (...)".

Estos tres principios se esbozaron en algunas de las prácticas y en los objetivos de los docentes a lo largo de la investigación. La integración y encastre del trabajo práctico y trabajo teórico, la innovación, el repensar la realidad actual, el rol de alumno a partir de su futuro papel como docente, son ejemplos que fuimos desarrollando a lo largo de este trabajo.

Ante el abandono del positivismo y el mecanicismo en ciencias, el auge de la Teoría de Sistemas, que se da en el último tercio del siglo .XX, toma forma en varias temáticas sociales, naturales y educativas. En el campo de la educación, se concibe al alumno como un sistema complejo, en el que confluye lo biológico, lo psicológico, lo social y lo cultural. El objeto de 
estudio se complejiza, ya que las ciencias abandonan el causalismo y la linealidad. El enseñante media y filtra los conocimientos, y el educando hace lo propio, no son un transmisor y una tabla rasa respectivamente. Son mediadores, constructores, creadores, modificadores, integradores.

Si definimos como un sistema a la conjunción alumno - profesor - laboratorio - conocimientos procedimentales, una propiedad emergente es la posibilidad de transferencia y generalización de los procedimientos aprendidos. Es una propiedad final que cumple el sistema, pero que no puede comprenderse ni explicarse por las propiedades y funciones individuales de sus componentes: no basta con leer en qué consiste el procedimiento ("tocar el piano con una sola mano"), o en ver cómo lo hace otro, o en lograr la experticia en el aula, o en buscar conexiones con la vida cotidiana y con otras áreas de las ciencias.

Aplicar, fuera del laboratorio, los procedimientos aprendidos:

Es necesario incluir contenidos científicos en la formación de cualquier estudiante, de cualquier nivel, más aun en formación docente, donde las personas que se están formando serán profesores de Ciencias. La duda que queda, cabalmente planteada por Hodson (1994) es: cuando se enseña en el laboratorio, ¿se hace de manera de que esos conocimientos sean transferibles y generalizables, ambas cualidades necesarias (pero no suficientes) para que los procedimientos aprendidos en el laboratorio de ciencias sean aplicados a la vida fuera del aula? Entonces, recurrimos a la cita de Henry Adams que hicieron propia dos excelentes autores: "Un profesor nunca logra saber dónde termina su influencia" (Philip W. Jackson -2002- en Práctica de la enseñanza y Stephen J. Gould -1986 - en El pulgar del panda). Los docentes se ven presionados para "buscar pruebas sólidas de que sus alumnos aprenden o no" pero quedan agobiados por "...las incertidumbres surgidas de su intención de alcanzar otras metas educativas." (Jackson 2002).

De acuerdo con la teoría del caos y al paradigma de la complejidad, se afirma que si bien el comportamiento de un sistema es impredecible, responde a un "orden subyacente" que parte de las condiciones iniciales del sistema (Romero 2006). Enseñar actitudes, valores y proporcionar pistas de la transferencia y generalización de los conocimientos procedimentales trabajados desde el laboratorio, más allá de si los alumnos los aprenden y los aplican, pareció ser para los docentes observados un objetivo primordial; pareció constituirse en una intención trascendente. Los profesores intentaron asegurar una condición inicial, aunque no tengan muchas formas de saber con certeza hasta qué punto estos contenidos serán tomados para la vida posteriormente.

Y este es el aspecto que rebosó los objetivos iniciales: la reflexión breve pero poderosa, acerca de qué manera la enseñanza de contenidos procedimentales puede ser útil más allá del laboratorio. Algunos de nosotros, como docentes, nos lo hemos planteado, otros no. Continuar tejiendo una respuesta, junto a algunos investigadores que lo han planteado, como Juan José Aparicio, Philip Jackson, Robin Millar et al., Stephen Jay Gould, entre otros, es el desafío que queda en este proceso que hemos intentado acercarles.

\section{Lo que vendrá:}

Escribe Stephen J. Gould (1997), en su obra "Milenio" que "Los poetas, cantando la interrelación de todas las cosas, dicen que la caída del pétalo de una flor perturbará una estrella lejana." Volviendo a la Teoría General de Sistemas, el Efecto Mariposa es el calificativo que se le da a "la dependencia sensible a las condiciones iniciales" que involucra un sistema complejo y que impactan su comportamiento volviéndolo impredecible (Romero 2006). El impacto de un trabajo cualitativo sobre la didáctica, en los cuerpos teóricos establecidos o en las prácticas de los docentes, no es fácil de presagiar, más cuando sabemos que no vamos a verificar presupuestos sino que lo que perseguimos es construcción de teoría, descubrimientos. 
Hallamos cuatro posibles áreas de impacto de este trabajo. En primer lugar, provocar la reflexión acerca de lo que se quiere y lo que se debe enseñar, con relación a los contenidos estrictos del currículo. ¿Se justifica dejar de ir al laboratorio porque no se tiene tiempo de terminar el programa? Hemos pretendido aportar elementos a esta reflexión, en el sentido de jerarquizar la enseñanza de procedimientos y valorar el ámbito del laboratorio como lugar privilegiado para ello. En segundo lugar, contribuir en lo que tiene que ver con el aprovechamiento de los recursos didácticos que existen en los laboratorios de los centros educativos y también con la generación de propuestas de trabajo, innovadoras y eficientes. El material de laboratorio es caro; este trabajo intentó indagar acerca de las posibilidades didácticas que ofrece un laboratorio bien equipado, y también de adecuarse a un posible contexto institucional diferente, innovando para lograr enseñar aunque se lo haga en un laboratorio "pobre". En tercer lugar, aspiramos a que la lectura de los resultados haga mirar hacia la influencia de la historia del docente, de la que muchas veces se derivan sus concepciones, las cuales se ponen en juego en el momento de decidir qué y cómo enseñar. Como dice Gaulejac (1987), todo individuo es producto y productor de la historia, y sólo el reconocimiento de esa realidad ineludible nos hace seres más libres, capaces, no de cambiar la historia sino de cambiar nuestra relación con ella. El aprendizaje de las ciencias de los futuros formadores, y la marca que ello imprime en la modalidad que éstos a su vez enseñarán en los centros de educación media, debería ser un aspecto a complementar en la temática que abordamos, y al cual esperamos impulsar aunque sea por medio de la reflexión. En cuarto lugar, queremos dirigir la atención hacia nuevas teorías científicas en las que enmarcar las prácticas docentes, modificando los esquemas teóricos dentro de los cuales podemos hallar fuentes de nuevas reflexiones y actitudes; nuevos motivos de inspiración.

La teoría de la complejidad nos provoca a tomar una postura epistemológica y filosófica en la que enmarcar la enseñanza de procedimientos, como una manera de darle sentido vital a la educación, de olvidarnos un poco de los resultados posibles y darle más trascendencia a los procesos. Y soñar con que los alumnos algún día tal vez sientan en su acción, la influencia de las enseñanzas de un antiguo profesor. Porque somos no sólo en la medida de lo que sabemos, sino también en la medida de lo que hacemos.

Fuimos testigos de cómo los docentes observados y entrevistados podían -pensar de forma autocrítica, -mejores formas de enseñar, a partir de su participación en este trabajo. Cuando el docente se siente analizado, si es un buen docente en el sentido moral, se analiza a sí mismo, se cuestiona, se critica. Por lo tanto, en el caso de que no hubiéramos perturbado el brillo de ninguna estrella, ni el vuelo de ninguna mariposa, aún queda para rescatar aquello que cambió desde nuestra postura frente a la enseñanza y aquello que desde nuestras preguntas e intercambios hayamos hecho reflexionar a los docentes investigados y a los lectores de este trabajo.

\section{Bibliografía:}

APARICIO, J. J. 1995. El conocimiento declarativo y procedimental que encierra una disciplina y su influencia sobre el método de enseñanza. En: Tarbiya, $n^{\circ} 10$ : pp. 23-38. Madrid: Instituto de Ciencias de la Educación, Universidad Autónoma de Madrid.

CAFFERATA, M.T. 2004. Una investigación sobre prácticas de laboratorio de biología en la escuela media. En: Memorias de las VI Jornadas Nacionales y I Congreso Internacional de la Enseñanza de la Biología. pp. 260-263. Buenos Aires: Editado por ADBIA.

CARRETERO, M. 1996. Construir y enseñar las ciencias experimentales. Buenos Aires: Editorial Aique. 
DE LA TORRE, S. y BARRIOS, O. 2000. (coords.). Estrategias didácticas innovadoras. Colección Recursos n $n^{\circ}$ 31. Barcelona: Ediciones Octaedro.

DE PRO BUENO, A. 1995. Reflexiones para la selección de contenidos procedimentales en ciencias. En: Alambique Didáctica de las Ciencias Experimentales n 6: pp. 77-87. Buenos Aires: Editorial Graó.

DIAZ BARRIGA, A. 1995. Didáctica. Aportes para una polémica. Buenos Aires: Aique Grupo Editor.

FELDMAN, D. 1999. Ayudar a enseñar. Relaciones entre didáctica y enseñanza. Colección Psicología Cognitiva y Educación. Buenos Aires: Editorial Aique.

FERNÁNDEZ, A. 1999. La inteligencia atrapada. Colección Alternativas en Salud Mental. $10^{\circ}$ ed. Buenos Aires: Ediciones Nueva Visión.

GAULEJAC, V. 1987. Historia e historicidad. En: La Névrose de classe. Trajectoire sociale e conflits d'identité. (Traducción de Lic. Argene Benedetti Sala, Montevideo). París: Editorial Homes y Groupes.

GOETZ, J. y LE COMPTE M. 1988. Etnografia y diseño cualitativo en investigación educativa. Madrid: Ediciones Morata.

GOODSON, I. y WALKER R. 1995. Contar cuentos. En: MC EWAN, H. y EGAN, K. (comps). La narrativa en la enseñanza, el aprendizaje y la investigación. Colección Agenda Educativa. Buenos Aires: Amorrortu Editores.

GOULD, S. J. 1986. El pulgar del panda. Buenos Aires: Editorial Hispamérica.

GOULD, S. J. 1993. Ocho cerditos. Reflexiones sobre historia natural. Barcelona: Editorial Crítica.

GOULD, S. J. 1997. Milenio. Barcelona: Editorial Crítica.

INSAUSTI, M. J. y MERINO, M. 2000. Una propuesta para el aprendizaje de contenidos procedimentales en el laboratorio de física y química. En: Investigações em ensino de ciencias on line. Vol. 5, N. 2, agosto de 2000. citado en enero de 2006. Porto Alegre: Universidade Federal do Rio Grande do Sul. Disponible en: http://www.if.ufrgs.br/public/ensino/vol5/n2/ 12indice.htm.

JACKSON, P. W. 2002. Práctica de la enseñanza. Colección Agenda educativa. Buenos Aires: Amorrortu Editores.

LITWIN, E. 1997. Las configuraciones didácticas. Una nueva agenda para la enseñanza superior. Buenos Aires: Editorial Paidos.

MASTACHE, A. 1998. Filosofía de las ciencias particulares y didácticas especiales. En: Revista del Instituto de Investigaciones en Ciencias de la Educación. Año VII, N¹3: pp: 15-18. Buenos Aires: Miño y Dávila Editores.

MEIRIEU, P. 1997. Aprender, sí. Pero ¿cómo? (2da. Edición). Barcelona: Editorial Octaedro. MILLAR R.; LE MARÉCHAL, J. F. y TIBERGHIEN, A. 1999. 'Mapping' the domain. Varieties of practical work. En: LEACH, J. y PAULSEN, A. C. Practical work in science education: recent research studies. pp: 33-59. Roskilde, Denmark: University of Roskilde University Press.

PARDINAS, F. 1999. Metodología y técnicas de investigación en Ciencias Sociales. $36^{\mathrm{a}}$ ed. México: Editorial Siglo XXI Editores. 
ROMERO, C. 2006. Paradigma de la complejidad, modelos científicos y conocimientos educativos. En: Revista Agora Digital. Universidad de Huelva. on line. citado en mayo de 2006. Disponible en: http://www.uhu.es/agora/digital/numeros/06/06articulos/monografico/pdf_6/ clara_romero.pdf.

SANMARTI, N., MAURI, T., IZQUIERDO, M. y GÓMEZ, I. 1990. Los procedimientos. Cuadernos de Pedagogía No 180. Montevideo: CODICEN.

SALGUEIRO, A.M. 1998. Saber docente y práctica cotidiana. Un estudio etnográfico. Barcelona: Editorial Octaedro.

SPIEGEL, A. 1999. Docente protagonista: docente compositor. Buenos Aires: Ediciones Novedades Educativas.

STAKE, R. 1999. Investigación con estudio de casos. Colección Pedagogía-Manuales. $2^{\circ}$ ed. Madrid: Ediciones Morata.

TAYLOR, S. y BOGDAN, R. 1987. Introducción a los métodos cualitativos de investigación. La búsqueda de significados. Colección Paidós Básica, № 37. Barcelona: Editorial Paidós.

VALLES, M. 1997. Técnicas cualitativas de investigación social. Reflexión metodológica y práctica profesional. Madrid: Editorial Síntesis.

WOODS, P. 1993. La escuela por dentro. La etnografía en la investigación educativa. Colección Temas de Educación. $3^{\mathrm{a}}$ Ed. Madrid y Barcelona: Coeditado por el Centro de Publicaciones del Ministerio de Educación y Ciencia de España y Ediciones Paidós.

ZABALA VIDIELLA, A. 2000. La práctica educativa. Cómo enseñar. Serie Pedagogía. Teoría y Práctica. $7^{\circ}$ ed. Barcelona: Editorial Graó.

*Master en Educación, Universidad ORT Uruguay. Diploma en Educación, Universidad ORT Uruguay. Licenciada en Ciencias Biológicas, Universidad de la República. Docente, Centro Regional de Profesores del Sur.

**Magister en Didáctica Universidad de Buenos Aires. Especialista en Didáctica, Universidad de Buenos Aires. Profesora en Ciencias Biológicas con especialización en Informática Educativa, Consejo Superior de Educación católica. Profesora en Ciencias Biológicas, Universidad Nacional de Córdoba. Tutora, Master en Educación, Universidad ORT Uruguay. Docente, Enseñanza Secundaria. 